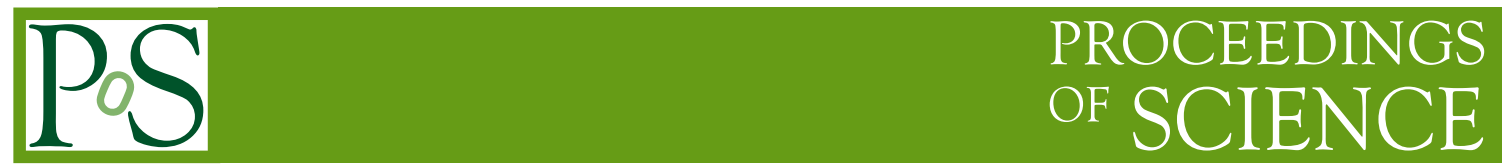

\title{
The electro weak transition and the equation of state in the SU(2)-Higgs-model
}

\author{
Jana Günther* \\ University of Wuppertal \\ E-mail: Jana. Guentheret-online.de
}

\begin{abstract}
Since the discovery of the Higgs particle at the LHC it is possible to investigate the equation of state for the electro weak transition in the $S U(2)$-Higgs-model at physical parameters. We will present a line of constant (LCP) physics and preliminary results on the equation of state for small $N_{t}$ values. The data was obtained by simulation with a combined heatbath and overrelaxation algorithm.
\end{abstract}

The 32nd International Symposium on Lattice Field Theory,

23-28 June, 2014

Columbia University New York, NY

${ }^{*}$ Speaker. 


\section{Introduction}

To describe the expansion of the early universe in the theory of the big bang it is important to understand the relation between the temperature and for example energy density and pressure. If these are known one can understand the temporal development of the universe from general relativity. For this reason we want to learn about the equation of state. In this work a first study of the equation of state for the electroweak transition in the $S U(2)$-Higgs-model is presented. In this model only the $S U(2)$ gauge fields and a scalar doublet are simulated. By using this model in 1996 the electro weak transition was determined to be a cross over [1]. In 1998 the critical endpoint was experimentally found to be at a Higgs mass of $(66.5 \pm 1.4) \mathrm{GeV}[2,3]$. Since in 2012 the Higgs boson was found to be $(125.9 \pm 0.4) \mathrm{GeV}[4]$ by the LHC it is possible to do simulations at the physical Higgs mass.

\section{Algorithms and observables}

In the Standard Model the electroweak interaction is described by the $S U(2) \times U(1)$ gauge symmetry. For simulations of the electroweak sector on the lattice the $U(1)$ degrees of freedom often are integrated out, while fermions and the strong interaction are not take into account in the construction of the action. This gives the continuum action

$$
S_{K}=\int \mathrm{d}^{4} x \frac{1}{4} \operatorname{tr} F_{\mu v} F^{\mu v}+D_{\mu} \phi D^{\mu} \phi+m_{\mathrm{K}} \phi_{\mu} \phi^{\mu}+\lambda_{\mathrm{K}}\left(\phi_{\mu} \phi^{\mu}\right)^{2} .
$$

Where $F$ is the field strength tensor of the $S U(2)$ gauge fields, $D$ is the covariant derivative and $\phi$ is a complex scalar doublet.

The gauge part of the action can be discretized by the standard $S U(2)$ wilson gauge action. The straight forward discretization of the scalar part of the action leads to

$$
S_{\mathrm{S}}=\sum_{x \in \Lambda} \sum_{\mu=1}^{4}\left(U_{x \mu} \phi_{x+\hat{\mu}}-\phi_{x}\right)^{\dagger}\left(U_{x \mu} \phi_{x+\hat{\mu}}-\phi_{x}\right)+m_{\mathrm{K}}^{2} \phi_{x}^{\dagger} \phi_{x}+\lambda_{\mathrm{K}}\left(\phi_{x}^{\dagger} \phi_{x}\right)^{2} .
$$

One now can reparametrize the action by introducing

$$
\begin{aligned}
\lambda_{\mathrm{K}} & =\frac{\lambda}{\kappa} \\
m_{\mathrm{K}}^{2} & =\frac{1-2 \lambda}{\kappa}-2 \\
\phi_{x} & =\sqrt{\kappa} \Phi_{x}
\end{aligned}
$$

and than write the scalar field as a matrix

$$
\Phi=\left(\begin{array}{c}
\Phi_{1} \\
\Phi_{2}
\end{array}\right) \longrightarrow\left(\begin{array}{cc}
\Phi_{2}^{*} & \Phi_{1} \\
-\Phi_{1}^{*} & \Phi_{2}
\end{array}\right)=\varphi
$$

This yields a suitable action for simulating the $S U$ (2)-Higgs-model

$$
\begin{aligned}
S[U, \varphi]=\beta \sum_{p l}(1- & \left.\frac{1}{2} \operatorname{tr} U_{p l}\right) \\
& +\sum_{x}\left(\frac{1}{2} \operatorname{tr}\left(\varphi_{x}^{\dagger} \varphi_{x}\right)+\lambda\left(\frac{1}{2} \operatorname{tr}\left(\varphi_{x}^{\dagger} \varphi_{x}\right)-1\right)^{2}-\kappa \sum_{\mu=1}^{4} \operatorname{tr}\left(\varphi_{x}^{\dagger} U_{x \mu} \varphi_{x+\mu}\right)\right) .
\end{aligned}
$$


The Simulation was carried out by using an heatbath algorithm described in [5] and an overrelaxation algorithm based on the description in [6]. While the heatbath algorithm guarantees the ergodicity of the update the overrelaxation algorithm is used to decrease the autocorrelation time. For comparison also an hybrid-monte-carlo algorithm was implemented.

Now the observables needed for the following analysis shall be introduced. The easiest observables are these which can be measured on every configuration. These are

$$
\begin{aligned}
R_{x} & =\operatorname{det} \varphi_{x}=\frac{1}{2} \operatorname{tr}\left(\varphi_{x}^{\dagger} \varphi_{x}\right) \\
L_{\varphi, x \mu} & =\frac{1}{2} \operatorname{tr}\left(\varphi_{x}^{\dagger} U_{x \mu} \varphi_{x+\hat{\mu}}\right) \\
P_{P l} & =1-\frac{1}{2} \operatorname{tr} U_{p l} \\
Q_{x} & =\left(\rho_{x}^{2}-1\right)^{2} \\
S_{x} & =6 \beta P_{P l}+R_{x}+\lambda Q_{x}-8 \kappa L_{\varphi, x \mu} .
\end{aligned}
$$

For the determination of the masses several correlation functions were evaluated. For the determination of the higgs boson mass the correlators form the observables $R_{x}$ and $L_{\varphi, x \mu}$ were used. The $\mathrm{W}$ boson mass was determined by the correlation functions described in [7] from the observables

$$
W_{x r k}^{(n)}=\frac{1}{2} \operatorname{tr}\left(\sigma_{r} \varphi_{x}^{\dagger} U_{x k} U_{x+\hat{k}, k} U_{x+2 \hat{k}, k} \ldots U_{x+(n-1) \hat{k}, k} \varphi_{x+n \hat{k}}\right)
$$

for $1 \leq n \leq 6$. The values for the masses were than extracted from an effective mass plot.

Finally for the determination of the LCP the renormalized coupling $g_{R}^{2}$ is needed. This can be be calculated form the static potential which is given in terms of the Wilson-Loops $\mathscr{W}$ as

$$
V(R)=-\lim _{T \rightarrow \infty} \frac{1}{T} \ln \mathscr{W}(R, T) .
$$

It can be fitted with the Ansatz

$$
V(R)=C-\frac{A}{R} e^{-M R}+D G(M, R)
$$

where $A, C, D$ and $M$ are fit parameters. $G(M, R)$ are lattice correlations as given in [8]. The fit parameter $M$ can be related to the screening mass as described in [8]. One than can get the renormalized gauge coupling as

$$
g_{R}^{2}=\frac{16}{3} \pi A
$$

\section{Line of constant physics}

For determining a LCP tow parameters have to be fixed. Here the renormalized gauge coupling $g_{R}^{2}$ and the ratio between the higgs and the W mass $R_{H W}=\frac{m_{H}}{m_{W}}$ have been chosen. A problem when doing simulations in the $S U(2)$-Higgs-model is to decide to which values one wants to tune. As shown in [9] the influence of the $U(1)$ degrees of freedom is small. Unfortunately this is not true for influence of the top quark. Since it is not possible to simulate an $S U(2)+$ fermion theory at the moment the influence of the quarks have to be included perturbatively. 
For the LCP shown here a preliminary matching procedure was applied: The effective potential derived in [10] was expanded for hight temperatures both in the standard and in the $S U$ (2)-Higgsmodel. Afterwards the coefficients were matched, assuming that $R_{H W}$ is at the standard model value. This yields a renormalized coupling of $g_{R} \approx 0.428$. Other ways of determining the parameters and a procedure to estimate the error caused by the matching are still under investigation.

The results for the LCP shown in 1 where simulated on a $32^{4}$ lattice. The parameters were tuned until the values for $R_{H W}$ and $g_{R}^{2}$ are within a $10 \%$ interval of the required value. This $10 \%$ interval is indicated by the light red area. The grey area shows the error on the experimental values for the higgs and $\mathrm{W}$ mass. $\beta$ is kept constant while $\lambda$ depends linear on $\kappa$.

From the mass plot (figure 1) the scale can be determined. The result is shown in figure 2. For comparison one can look at the electroweak scale that is $0.0008 \mathrm{fm}$ or $246 \mathrm{GeV}$ [4].

\section{Equation of state}

After the determination of the LCP the equation of state can be calculated. Here the interaction measure $I$, the pressure $p$, the energy density $\varepsilon$ and the entropy $s$ will be calculated. The derivation of these quantities can be found in [11].

First the interaction measure has to be calculated. It can be expressed in terms of simple observables

$$
\begin{aligned}
I & =-\frac{N_{t}^{3}}{N_{s}^{3}} m_{H}\left(\frac{\partial \ln Z}{\partial \beta} \frac{\partial \beta}{\partial \kappa} \frac{\mathrm{d} \kappa}{\mathrm{d} m_{H}}+\frac{\partial \ln Z}{\partial \lambda} \frac{\partial \lambda}{\partial \kappa} \frac{\mathrm{d} \kappa}{\mathrm{d} m_{H}}+\frac{\partial \ln Z}{\partial \kappa} \frac{\mathrm{d} \kappa}{\mathrm{d} m_{H}}\right) \\
& =-N_{t}^{4} m_{H}\left(-6\left\langle P_{\mathrm{Pl}}\right\rangle \frac{\partial \beta}{\partial \kappa} \frac{\partial \kappa}{\partial m_{H}}-\langle Q\rangle \frac{\partial \lambda}{\partial \kappa} \frac{\partial \kappa}{\partial m_{H}}+8\left\langle L_{\varphi}\right\rangle \frac{\partial \kappa}{\partial m_{H}}\right) .
\end{aligned}
$$

The interaction measure can be expressed in simple derivatives of the partition sum $Z$. Since $\beta$ is constant the first term vanishes. Next the pressure has to be calculated. It is given as

$$
\begin{aligned}
\frac{p}{T^{4}} & =N_{t}^{4} \int_{\left(\kappa_{0}, \beta_{0}, \lambda_{0}\right)}^{(\kappa, \beta, \lambda)} \mathrm{d}(\kappa, \beta, \lambda)\left(\frac{1}{N_{t} N_{s}^{3}}\left(\begin{array}{c}
\frac{\partial \ln Z}{\partial \kappa} \\
\frac{\partial \ln Z}{\partial \beta} \\
\frac{\partial \ln Z}{\partial \lambda}
\end{array}\right)\right) \\
& =N_{t}^{4} \sum-6 \Delta \beta\left\langle P_{\mathrm{P} 1}\right\rangle-\Delta \lambda\langle Q\rangle+8 \Delta \kappa\left\langle L_{\varphi}\right\rangle .
\end{aligned}
$$

For renormalization the values of the zero temperature simulation are subtracted from the finite temperature results. Since the simulation is done at a high energy it is not reasonable to assume that the pressure vanishes for the smallest simulated energy to fix the integration constant. Instead it was assumed that for the largest simulated energy the value of the pressure equals the StefanBoltzmann-limit.

From the interaction measure and the pressure the energy and the entropy density can be calculated as

$$
\begin{aligned}
& \frac{\varepsilon}{T^{4}}=I+\frac{3 p}{T^{4}} \\
& \frac{s}{T^{3}}=\frac{\varepsilon}{T^{4}}+\frac{p}{T^{4}} .
\end{aligned}
$$

The results for all four quantities are shon in figure 3 for three different lattice sizes. 


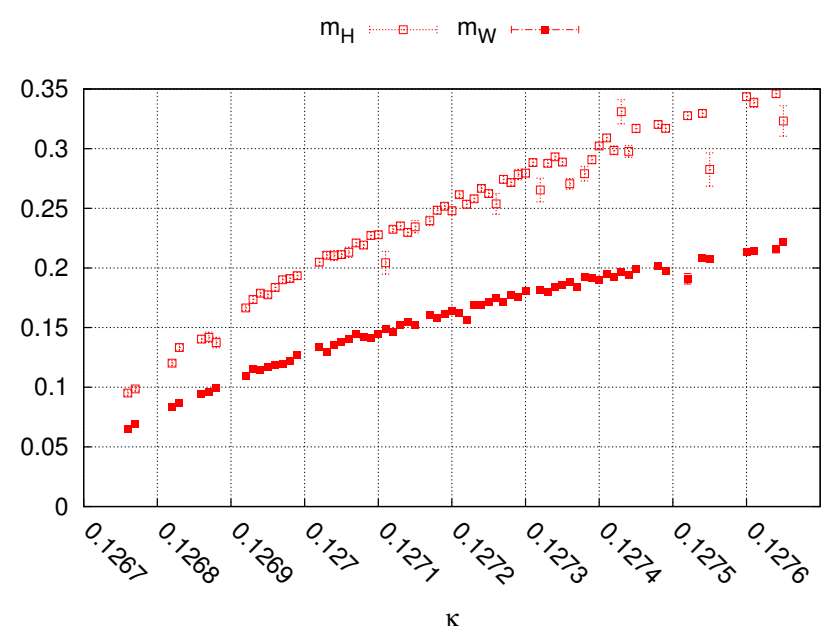

physical $R_{H W}$

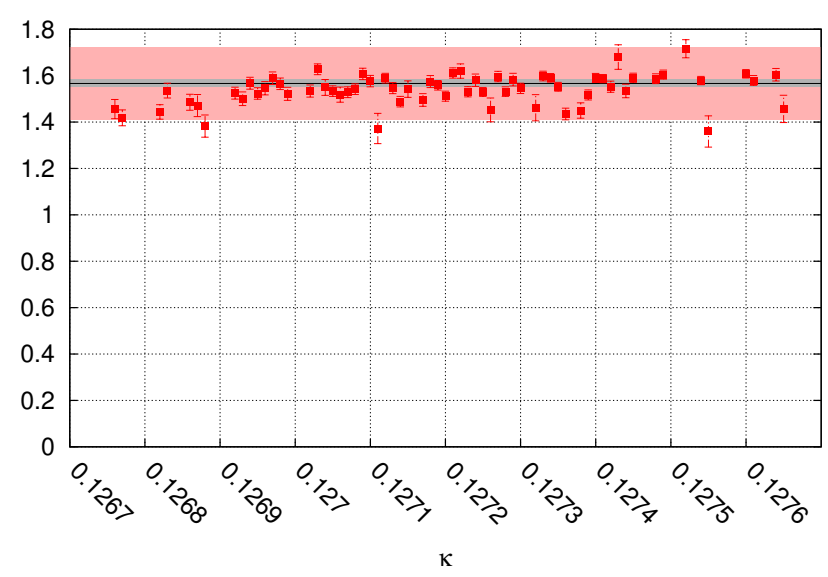

$g_{R}{ }^{2}$

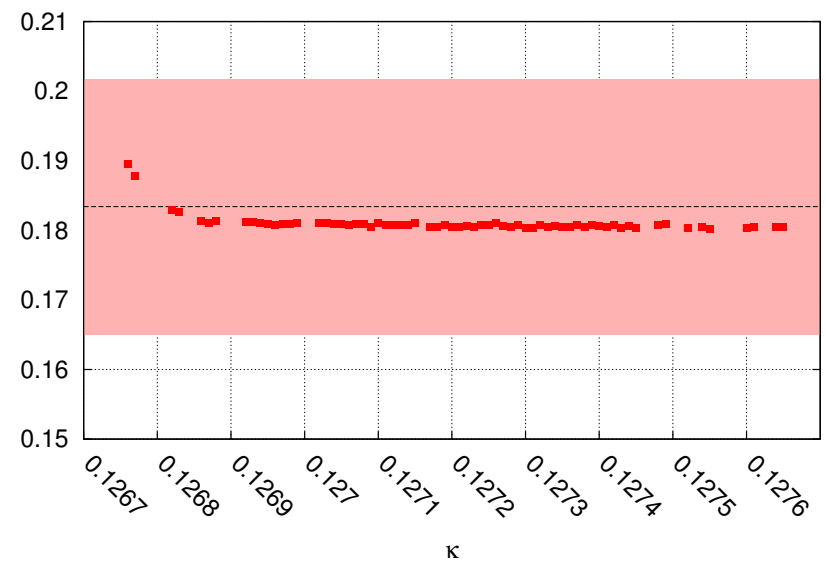

Figure 1: The LCP on a $32^{4}$ lattice. The light red are indicates the $10 \%$ interval in which the values are tuned. The grey are indicates the experimental error. 


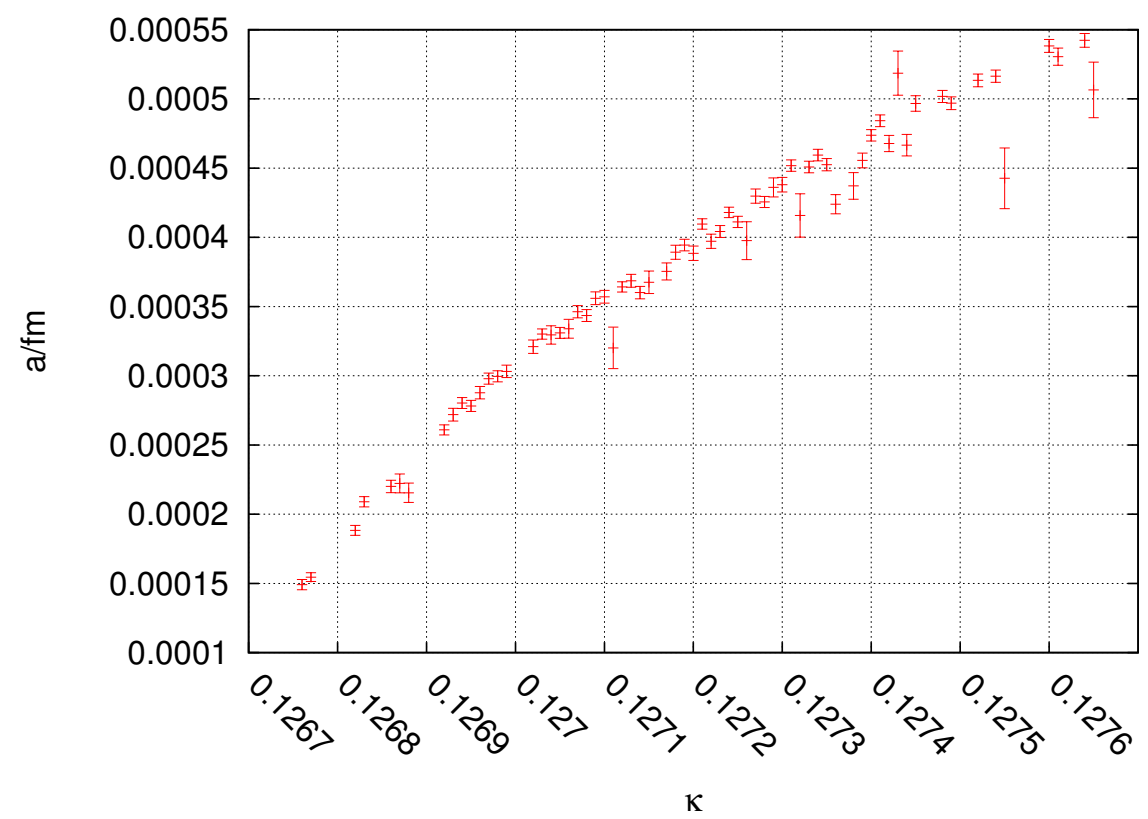

Figure 2: The scale at which the LCP was simulated. The electroweak scale is $0.0008 \mathrm{fm}$.
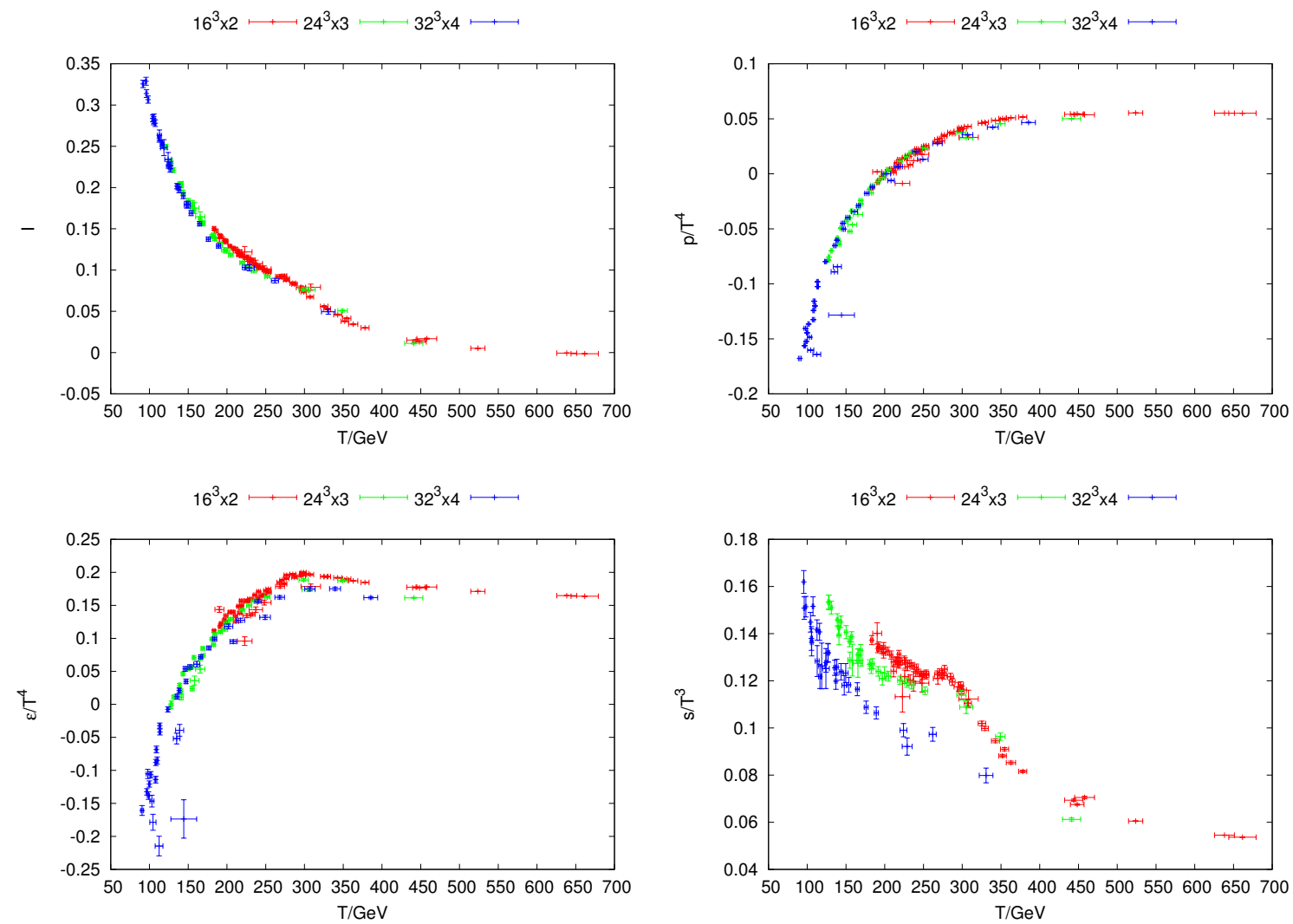

Figure 3: The results for the interaction measure $I$, the pressure $p$, the energy density $\varepsilon$ and the entropy $s$. 


\section{Summary}

In this work a preliminary result for an LCP trying to match the standard model at physical higgs mass and the resulting equation of state for the interaction measure, the pressure, the energy and entropy density were presented. The calculation were carried out in the $S U(2)$-Higgs-model. The LCP was determined on a $32^{4}$ lattice. The equation of state was calculated on the three lattice sizes $16^{3} \times 2,24^{3} \times 3$ and $32^{3} \times 4$. Larger lattices would be interesting. Therefore parameter tuning to finer lattices are needed to be able to investigate the interesting region of the electroweak transition. Also other ways to determine the values for tuning the LCP and an estimate for the error resulting from using the $S U(2)$-Higgs-model are still under investigation.

\section{Acknowledgement}

I would like to thank Zoltán Fodor, Christian Hölbling and Szabolcs Borsányi for the stimulating suggestions and continuous support throughout the project. This work is supported in part by the DFG grant SFB/TR55 and the GSI grant WF1012. (other numbers)

\section{References}

[1] K. Kajantie, M. Laine, K. Rummukainen and M. Shaposhnikov, Electroweak Phase Transition at $m_{H} \gtrsim m_{W}$ ?, Phys. Rev. Lett 77 (1996) 2887 [arXiv: 9605288 [hep-ph] ].

[2] Z. Fodor, F. Csikor, J. Heitger, Y. Aoki and A. Ukawa, End point of the electroweak phase transition, in Copenhagen 1998, Strong an electroweak matter (1998) 190 [arXiv: 9901307 [hep-ph]].

[3] Y. Aoki, F. Csikor, Z. Fodor, and A. Ukawa, The endpoint of the first order phase transition of the SU(2) gauge - Higgs model on a four-dimensional isotropic lattice, Nucl. Phys. Proc. Suppl. 73 (1999) 656 [arXiv: 9809122 [hep-lat] ].

[4] Particle Data Group Kollaboration, J. Beringer et al., Review of particle physics, Phys. Rev. D 86 (2012) 010001.

[5] Z. Fodor, J. Hein, K. Jansen, A. Jaster and I. Montvay, Simulating the electroweak phase transition in the SU(2) Higgs model, Nucl. Phys. B439 (1995) 147 [arXiv: 9409017 [hep-lat] ].

[6] Z. Fodor and K. Jansen, Overrelaxation algorithm for coupled gauge Higgs systems, Phys. Lett. B331 (1994) 119 [arXiv: 9403024 [hep-lat]].

[7] B. Bunk, E.-M. Ilgenfritz, J. Kripfganz and A. Schiller, The finite-temperature phase transition in lattice SU(2) Higgs theory at weak couplings, Nucl. Phys. B403 (1993) 119.

[8] W. Langguth, I. Montvay and P. Weisz, MONTE CARLO STUDY OF THE STANDARD SU(2) HIGGS MODEL, Nucl. Phys. B277 (1986) 11.

[9] K. Kajantie, M. Laine, K. Rummukainen and M. Shaposhnikov, A non-perturbative analysis of the finite T phase transition in the $S U(2) \times U(1)$ electroweak theory, (1996) [arXiv: 9612006 [hep-ph]].

[10] Z. Fodor and A. Hebecker, Finite temperature effective potential to order $g^{4}, \lambda^{2}$ and the electroweak phase transition, Nucl. Phys. B432 (1994) 127 [arXiv: 9403219 [hep-lat]].

[11] Z. Fodor and S. Katz, The Phase diagram of quantum chromodynamics, (2009) 